\title{
Propagating Neocortical Gamma Bursts Are Coordinated by Traveling Alpha Waves
}

\author{
Ali Bahramisharif, ${ }^{1}$ Marcel A. J. van Gerven, ${ }^{1}$ Erik J. Aarnoutse, ${ }^{2}$ Manuel R. Mercier, ${ }^{3}$ Theodore H. Schwartz, ${ }^{4}$ \\ John J. Foxe, ${ }^{3}$ Nick F. Ramsey, ${ }^{2}$ and Ole Jensen ${ }^{1}$ \\ ${ }^{1}$ Donders Institute for Brain, Cognition and Behaviour, Radboud University Nijmegen, 6525EN, Nijmegen, The Netherlands, ${ }^{2}$ Rudolf Magnus Institute, \\ Utrecht University, 3584CX, Utrecht, The Netherlands, ${ }^{3}$ The Sheryl and Daniel R. Tishman Cognitive Neurophysiology Laboratory, Children's Evaluation \\ and Rehabilitation Center, Department of Pediatrics and Neuroscience, Albert Einstein College of Medicine, Bronx, New York 10461, and ${ }^{4}$ Department of \\ Neurological Surgery, Weill Cornell Medical College, New York Presbyterian Hospital, New York, New York 10021
}

Neocortical neuronal activity is characterized by complex spatiotemporal dynamics. Although slow oscillations have been shown to travel over space in terms of consistent phase advances, it is unknown how this phenomenon relates to neuronal activity in other frequency bands. We here present electrocorticographic data from three male and one female human subject and demonstrate that gamma power is phase locked to traveling alpha waves. Given that alpha activity has been proposed to coordinate neuronal processing reflected in the gamma band, we suggest that alpha waves are involved in coordinating neuronal processing in both space and time.

\section{Introduction}

Cortical traveling waves have been investigated by intracranial recordings in several species, including turtle, rabbit, cat, dog, and monkey (for review, see Ermentrout and Kleinfeld, 2001; Sato et al., 2012). Traveling waves have also been observed in the scalp EEG, although there is some debate as to whether it reflects the propagation of cortical activity or if it can be explained by volume conduction of the activity from a few neuronal sources (Hughes, 1995; Nunez et al., 2001; Alexander et al., 2013). Recent work has demonstrated cross-frequency interactions in which the amplitude of gamma band activity is coupled to the phase of alpha activity (Osipova et al., 2008; Voytek et al., 2010; Spaak et al., 2012). Here, we investigated whether gamma activity is also linked to the phase of alpha oscillations traveling over cortex. If so, this would create a scenario in which bursts of gamma are propagating with the phase of the alpha wave. Electrocorticography (ECoG) allows us to measure neocortical activity with high temporal and spatial resolution. The relatively high signal-tonoise ratio obtained with ECoG makes it possible to reliably detect alpha and gamma activity and to investigate their spa-

Received June 7, 2013; revised Sept. 18, 2013; accepted Oct. 24, 2013.

Author contributions: A.B., M.A.J.v.G., and 0.J. designed research; A.B., M.A.J.v.G., E.J.A., M.R.M., T.H.S., J.J.F., N.F.R., and 0.J. performed research; A.B. analyzed data; A.B., M.A.J.v.G., and 0.J. wrote the paper.

A.B., MA.J.v.G., N.F.R., and 0.J. were supported by the BrainGain Smart Mix Programme of the Netherlands Ministry of Economic Affairs and the Netherlands Ministry of Education, Culture and Science. O.J. was supported by the Netherlands Organization for Scientific Research (NOW; VICI Grant 453-09-002) and "The Healthy Brain" funded by the Netherlands Initiative Brain and Cognition (Grant 056-14-01). E.J.A. and N.F.R. were supported by the Dutch Technology Foundation STW, the Applied Science Division of NOW, the Technology Program of the Ministry of Economic Affairs, and the University of Utrecht (Grant UGT7685).

The authors declare no competing financial interests.

Correspondence should be addressed to Ali Bahramisharif, Kapittelweg 29, 6525 EN, Nijmegen, The Netherlands. E-mail: a.bahramisharif@fcdonders.ru.nl.

DOI:10.1523/JNEUROSCI.2455-13.2013

Copyright $\odot 2013$ the authors $\quad 0270-6474 / 13 / 3318849-06 \$ 15.00 / 0$ tiotemporal interactions (Freeman et al., 2000; Osipova et al., 2008; Gunduz et al., 2011).

\section{Materials and Methods}

Experimental procedures. ECoG data collected from seven participants was used. The experimental design was resting state with either closed or open eyes. There were two groups of participants of either sex. Group 1 included participant numbers 1,2, 3, 5, and 6, who had their eyes closed during the experiment. Group 2 included participant numbers 4 and 7 , who were fixating on a blue "O" in the center of the screen that was located $\sim 100 \mathrm{~cm}$ ahead. The length of the experiment was between 3 and $12 \mathrm{~min}$. The sampling rate of the recording of data for Group 1 was 1000 $\mathrm{Hz}$ and for Group 2 was $512 \mathrm{~Hz}$. Data for Group 1 were collected in New York and data for Group 2 were collected in Utrecht, The Netherlands. The local Ethics Committee approved all relevant aspects of the experimental procedure. All datasets were provided completely anonymously. Data analyses were performed using MATLAB R2009 (MathWorks), either with custom-written scripts or with the FieldTrip toolbox (Oostenveld et al., 2011).

Offline data preprocessing and calculation of the spectra. To remove line noise, a notch filter was used with a band-stop frequency of $58-62 \mathrm{~Hz}$ for data from Group 1 and $48-52 \mathrm{~Hz}$ for data from Group 2. Next, a common average referencing filter was applied in which all measurement electrodes except the noisy ones were included. Electrodes above the neocortical areas that were considered epileptic and later resected were excluded from further analysis. Furthermore, noisy electrodes were the ones that showed a difference between the maximum and minimum raw voltage over all trials above a subjective threshold.

Frequency analysis was required for computing phase-locking values and phase-amplitude coupling. To estimate instantaneous phase and amplitude, we band-pass filtered the data. We used the magnitude and angle of the Hilbert-transformed band-pass-filtered data as our amplitude and phase estimates, respectively. We applied linear phase, finite impulse response filters. The filter orders were frequency dependent (order $=$ number of cycles $\times$ sampling frequency/frequency of interest) as well as the pass-band, which was $[f-f / 4, f+$ $f / 4]$ for the phase estimation and $[f-f / 8, f+f / 8]$ for the amplitude estimation, where $f$ is the frequency of interest. This resulted in a filter 
kernel that linearly decreased in length with frequency. When estimating the phase of the slower oscillations, we used three cycles; when estimating the amplitude of the faster oscillations, we used six cycles. These filter settings were used for calculating phase-amplitude couplings and phase-locking values.

Bartlett's method was used to calculate the power spectrum of $2-20 \mathrm{~Hz}$ (Bartlett, 1948). For each frequency band, in each window of data, 5 cycles were used, and the centers of windows were located $2.5 \mathrm{~s}$ apart. We chose to adapt the time window with frequency because the spectral smoothing increases with increasing frequency as for the filters used for the phase amplitude coupling. However, the results were similar when Welch's method was used. For each subject, the spectrum was first log transformed and then averaged over all windows and non-noisy electrodes.

Phase-locking value. To estimate the phase-locking values (PLVs), the average difference between the phases of different electrodes over the course of the experiment was calculated (Lachaux et al., 1999). The PLV quantifies the consistency in phase difference between two electrodes over trials.

Phase amplitude coupling. For each subject, we calculated the coupling of the power of different frequency bands of each electrode and the phase of the same electrode. To calculate the phase-amplitude coupling, for each phase and power frequency, the distance between the power distribution for each phase and the uniform distribution was calculated as the phase-amplitude coupling index (Tort et al., 2010). To correct the phase-amplitude coupling for noise, we divided the time length of the experiment into two equal parts. Then, we swapped the power of the first and the second parts and estimated the coupling with the original phase distributions. After that, we subtracted the coupling values obtained after swapping from the original coupling values. We then set all the negative values to zero. Subject-specific alpha and gamma central frequencies were selected based on the peak in the phase-amplitude coupling of the most posterior ventral electrode in each subject.

Spatial traveling waves. Traveling waves are observed with respect to a reference location. To identify the reference locations, we performed the following analysis: for a putative reference location, motifs of traveling waves were identified. A motif consisted of four or more consecutive electrodes (horizontal or vertical gridwise directions) in which there was a systematic phase advance (or decay) of $<90$ degrees between neighboring electrodes. Further, only electrodes with a PLV $>0.1$ with respect to the putative reference location were considered. We then counted the number of motifs that a given electrode participated in with respect to all reference locations. The resulting counts are shown in Figure 3.

To identify the optimal reference location, we considered the PLV between a putative location and the motif with the highest count (summed over members in a motif). The chosen reference location was the one in which the PLV was the highest for the dominating motif (for each motif, we considered the PLV values of the 4-5 members; the lowest of these was considered the characterizing PLV value of that motif). Further, this PLV was used as the threshold for the significance test.

To assess the robustness of the traveling waves statistically, we performed a permutation test. The null hypothesis was that the phase advance (decay) of the identified traveling wave occurred by random. For example, when testing a motif of five electrodes, we first identified the electrodes exceeding the PLV threshold (see previous paragraph). amplitude.

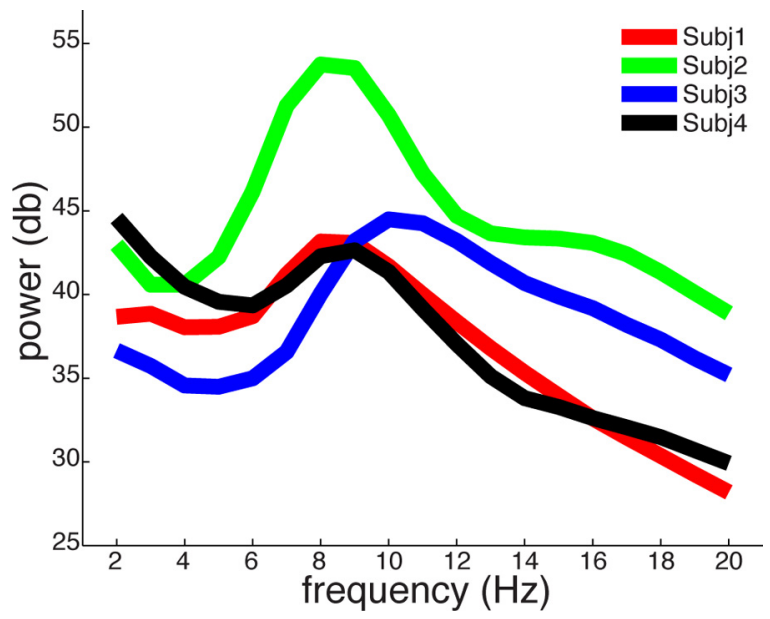

Figure 1. Power spectra averaged over all trials and electrodes for each participant. The analysis reveals dominant peaks in the alpha band $(8-10 \mathrm{~Hz})$.
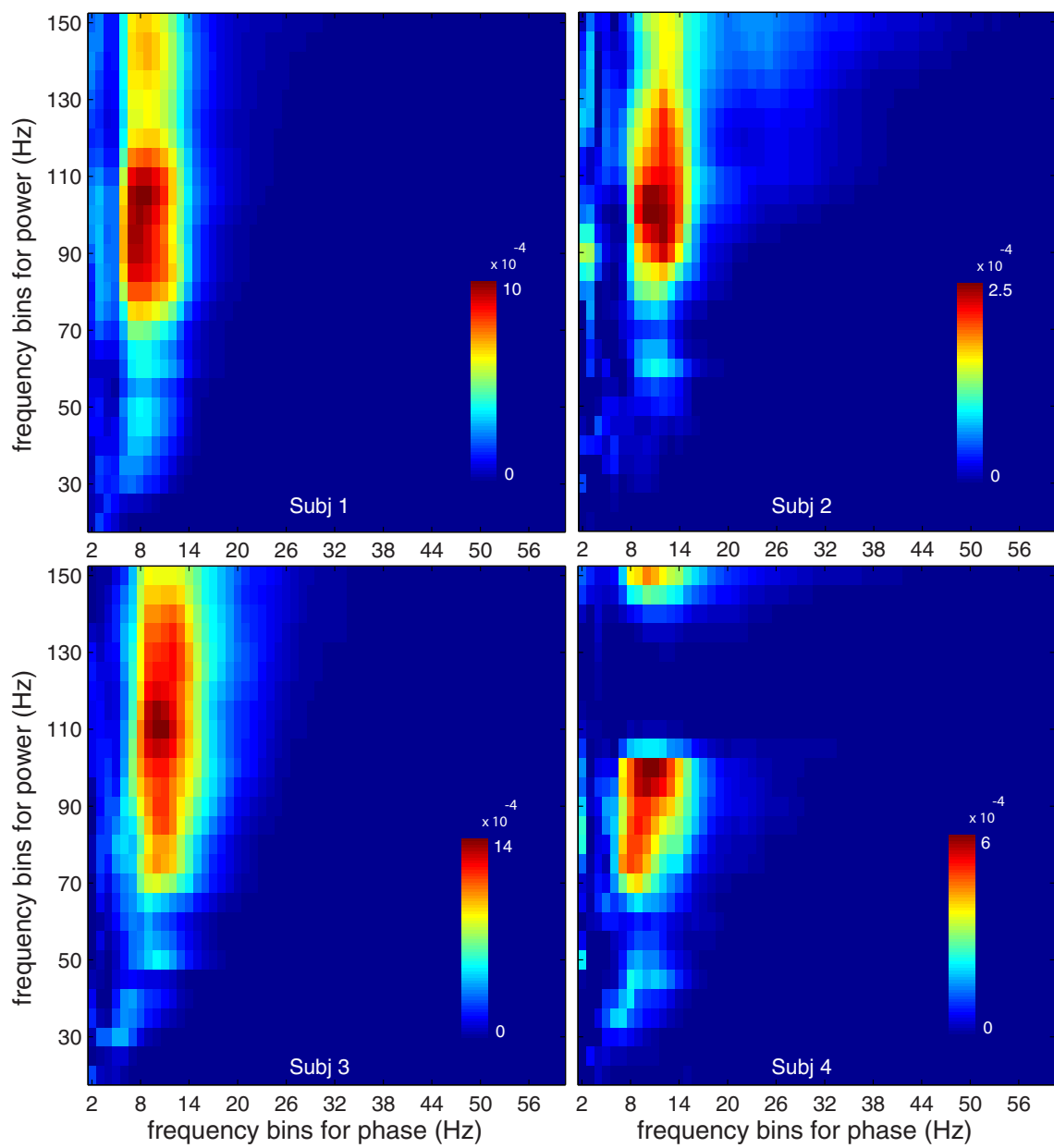

Figure 2. Phase-amplitude coupling over the most posterior ventral electrodes. The color scale reflects the degree of nonuniformity of the distribution of high-frequency amplitude ( $y$-axis) with respect to the phase of the low-frequency oscillations $(x$-axis). Note that the coupling was constrained to the alpha band with respect to phase and high gamma band with respect to
Then, we permuted the phase values for these locations and counted how many times the systematic phase advance (or decay) occurred for a group of at least five consecutive electrodes. The number of occurrence divided by the permutations $(N=1000)$ constituted the $p$-value. 


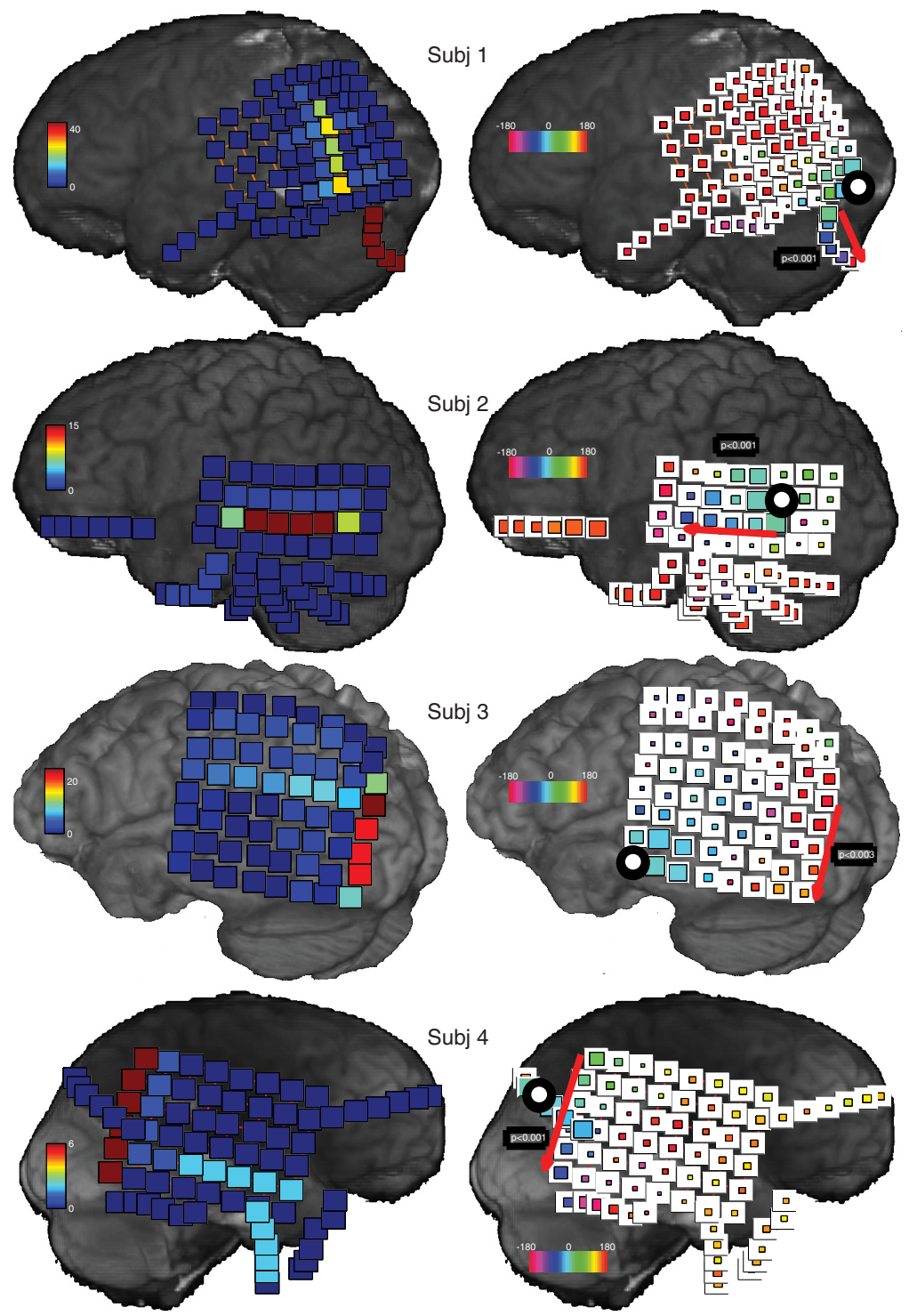

Figure 3. Left: Number of times that each group of electrodes (a motif) showed the traveling wave pattern for all possible reference locations. Right: Average alpha phase difference between all electrodes and the reference location (white disc). The color code shows the phase difference ( -180 to 180). The size of each square shows the magnitude of the PLV between a given electrode and the reference. The red arrow indicates a linear arrangement of electrodes where a systematic phase difference was observed (i.e., a traveling wave). The $p$-values refer to the statistical significance of systematic phase advances (or decays).

To plot the traveling pattern, the raw time series of the reference location were filtered in the band of $8-12 \mathrm{~Hz}$ using a Butterworth infinite impulse response filter of the fourth order. Then, the peaks of the bandpass-filtered reference location were detected. For the selected electrodes, the average of either the raw time series or the gamma power of windows of $300 \mathrm{~ms}$ around the alpha peaks of the reference location was calculated. The gamma power was calculated using a window of $100 \mathrm{~ms}$ centered at each time point with a $5 \mathrm{~ms}$ time step. The average velocity of travel was calculated by dividing the difference of the peak location of first and the last oscillators by the physical distance between them.

\section{Results}

ECoG datasets were analyzed from seven participants during the resting state. Four of the subjects showed a peak in the alpha band in the power spectrum and we exclusively focus the analysis on these subjects. Three of the subjects had their eyes closed and one (Subject 3) was fixating on a blue "O" on a screen located $\sim 100$ $\mathrm{cm}$ ahead.

\section{Gamma power is phase locked to alpha oscillations}

The power spectra averaged over all trials and electrodes of the four participants are shown in Figure 1. Note the prominent peaks in the alpha band ranging from 8 to $10 \mathrm{~Hz}$. On average, $330 \mathrm{~s}$ of data was used per subject.

To characterize the coupling between the alpha band activity and activity in the other frequency bands, we calculated the modulation index for phase-amplitude coupling (PAC) as developed by Tort et al. (2010). This measure quantifies the distribution of power in a given band with respect to the phase of activity in a lower-frequency band. A highly nonuniform power distribution suggests phase-amplitude coupling. This PAC measure was calculated for each electrode. Figure 2 shows that there is a strong coupling between alpha phase and gamma power in the most posterior-ventral electrode in all subjects. As can be seen in Figure 2, subject-specific dominant alpha frequencies were $8,11,10$, and $11 \mathrm{~Hz}$ and subject-specific dominant gamma modulation frequencies were 105, 100, 110, and 100 $\mathrm{Hz}$, for Subjects 1-4, respectively.

\section{Alpha waves travel over neocortex}

Traveling waves were quantified with respect to a reference location. By changing the reference location, different traveling wave patterns can be observed. Figure 3, left, shows the number of times that each group of electrodes (a motif) showed the traveling wave pattern when changing the location of the reference. Results indicate that traveling wave patterns were relatively independent of the reference location.

For each participant, the reference location was chosen to be the one with the highest coupling with the motif with the highest counts (white disc). From the PLVs, we then derived the difference in alpha phase with respect to the reference location (phase difference indicated by color in Fig. 3, right). The maximum PLVs were $0.72,0.71,0.58$, and 0.55 for Subjects $1-4$, respectively. Different traveling directions were observed for different participants.

\section{Gamma bursts propagate with the alpha waves}

For the arrays of electrodes identified in Figure 3, we investigated the traveling wave phenomenon in more detail. Figure 4 shows at least 1500 averaged raw traces for either five or six consecutive electrodes. Before averaging, each trace was phase aligned according to the alpha band activity in the reference location (i.e. "peak aligned") and then mean corrected. To detect the peaks, the signal from the reference location was band-pass filtered in the subject-specific alpha band. The average traces were normalized such that the amplitude per electrode was one. The plots show systematic changes in alpha phase over electrodes reflecting 
a traveling wave. The average velocities of traveling waves were $0.73,1.6,2.05$, and $1.85 \mathrm{~m} / \mathrm{s}$ for Subjects 1-4, respectively.

For the phase-aligned raw traces, we also calculated the gamma band power over time using a $100 \mathrm{~ms}$ sliding time window. The average traces were mean corrected and normalized. The analysis revealed that the gamma power was phase aligned according to the local phase of the alpha oscillations. This illustrates that the gamma bursts systematically travel with the phase of the propagating alpha wave. To further quantify this observation, we calculated the phase difference between the signal from the reference location and the other electrodes. This was done for the individual peak alpha frequencies for at least 1500 traces. The distribution of phase differences for Subject 1 is shown in the rose plots in Figure 4, blue. Next, we quantified the phase difference between the alpha band activity in the reference location and the temporal evolution of gamma power in the other electrodes (Fig. 4 , rose plots, green). To better visualize the distribution of gamma power with respect to alpha phase, we subtracted the minimum gamma amplitude of the phase distribution from all of the values in the phase distribution. Note that the gamma band power is in antiphase with the alpha activity. This analysis confirmed the occurrence of the gamma bursts at the troughs of the traveling alpha band activity.

\section{Discussion}

Based on data from four participants with implanted ECoG grids covering posterior neocortical regions, we demonstrate cases of gamma bursts propagating over neocortex with a velocity of $0.7-2.1 \mathrm{~m} / \mathrm{s}$. The gamma bursts were phase locked to traveling alpha waves and occurred at the troughs of the local alpha oscillations.

We propose that traveling alpha waves coordinate neuronal processing in time and space as reflected in gamma band activity. This proposal is based on several recent observations. First, numerous studies have now established that the phase of theta and alpha oscillations is locked to gamma band activity. This was first demonstrated in rat recordings (Bragin et al., 1995) and has more recently been demonstrated in monkey and human data (Lakatos et al., 2005; Canolty et al., 2006; Osipova et al., 2008; Sauseng et al., 2009; Canolty and Knight, 2010; Voytek et al., 2010; Spaak et al., 2012). Whereas gamma band activity has been associated with neuronal processing (Fries et al., 2007; Jensen et al., 2007; Siegel et al., 2012), alpha band activity is typically associated with top-down control (Klimesch et al., 2007b; Foxe and Snyder, 2011; Jensen et al., 2012). The coordination of gamma band activity by traveling alpha waves could play an important computational role. It should be emphasized that alpha band activity typically reflects pulses of inhibition. Therefore,
Subj 1
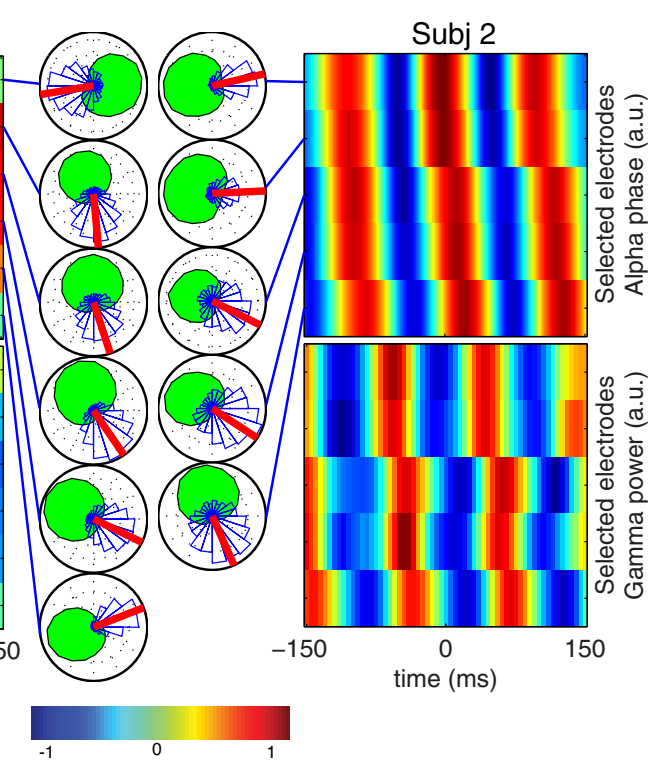

Subj 3
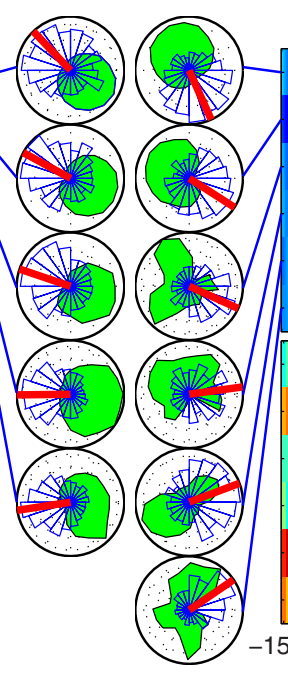

Subj 4

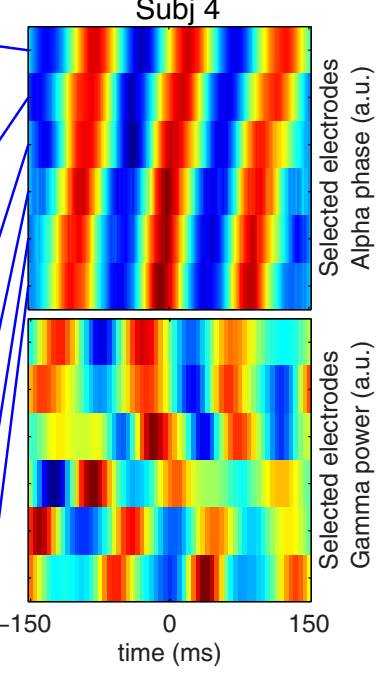

Figure 4. Top: Phase aligned to the alpha activity in the reference location and either five or six consecutive electrodes (Fig. 3). Note that the averaged traces are not band-pass filtered, but they are normalized to have zero mean and SD one. Bottom: Normalized temporal evolution for the high gamma band. The rose plots show the distribution of alpha phase (in blue) corresponding to zero phase of the reference location, and the red line shows the mean. The green regions show the gamma power distribution corresponding to the zero phase of the reference location.

traveling alpha waves in the visual system might serve to leave parts of the visual field unresponsive by preventing gamma band synchronization, and the traveling waves will create sweeps scanning the visual field (Ermentrout and Kleinfeld, 2001). This mechanism is consistent with the searchlight hypothesis (Crick, 1984). Traveling waves might also be involved in more intricate coding schemes. In rats, there is strong evidence that phasespecific firing at the theta cycle carries different information (O'Keefe and Recce, 1993; Lisman and Jensen, 2013). Likewise, alpha oscillations in the visual system have been proposed to coordinate a phase code. It is possible that the traveling waves could serve to tag visual features to different phases of the oscillations (Ermentrout and Kleinfeld, 2001). According to this scheme, simultaneously activated retinotopic visual representations would be organized in a temporal code coordinated by propagating alpha waves. Further experimental work linking perception and single unit firing to traveling waves is required to test these hypotheses. 
It has been shown that the traveling waves are absent in the presence of certain experimental paradigms (Ermentrout and Kleinfeld, 2001). Specifically in the visual cortex, traveling waves are mostly engaged when visual stimuli are weak or absent (Sato et al., 2012). In this report, we had three subjects with eyes closed and one with eyes open. We did not observe any systematic difference between the two groups. Indeed, all of them showed the same consistent pattern of traveling waves, as can be seen in Figure 4.

Several mechanisms could account for the propagation of the gamma bursts coordinated by the alpha waves. One possibility is that a given cortical region serves as an alpha rhythm pacemaker. At a certain phase, the network is sufficiently disinhibited and a gamma burst will occur. The gamma burst will then propagate through excitatory connections (Sato et al., 2012). A second mechanism would rely on coupled alpha oscillators extending over neocortex. Through synaptic interactions, these oscillations could self-organize into traveling waves, allowing for propagating gamma bursts (Ermentrout and Kleinfeld, 2001). Finally, traveling waves might be coordinated by thalamic generators (Llinás et al., 1998). The pulvinar is known to generate alpha activity that is phase locked to neocortical areas (Lopes da Silva, 1991; Saalmann and Kastner, 2011; Saalmann et al., 2012). Multielectrode recordings in neocortex and thalamus paired with selective regional ablation of neuronal activity would be required to distinguish between these mechanisms.

One might be concerned that traveling waves are a consequence of volume conduction. Two alpha generators at different locations oscillating at different phases could by volume conduction produce traveling wave phenomena in an array of recording electrodes. Although this might be the case for scalp EEG recordings (Nunez and Srinivasan, 2006), it is not likely to explain traveling waves in the ECoG recordings. Cooper et al. (1965) have shown that electrodes in neuronal tissue a few millimeters away from a stimulation electrode usually show no sign of after discharges. Furthermore, recordings from rabbits showed distinct local differences in phase and shape of neuronal potentials in electrodes $1 \mathrm{~mm}$ apart (Petsche and Rappelsberger, 1970). The propagating gamma bursts confirm that volume conduction cannot explain the reported findings. The gamma bursts observed at the different electrodes occur at different instances in time. They can therefore only be explained by activations of different local populations.

The velocity of travel that we observed was between 0.7 and 2.1 $\mathrm{m} / \mathrm{s}$. The literature is not clear on traveling speed of oscillatory waves. A study in dogs by Lopes da Silva reported a velocity of $\sim 0.3 \mathrm{~m} / \mathrm{s}$ (Lopes da Silva, 1991). This is consistent with the propagation speed of evoked nonrhythmic waves in several animal preparations (Sato et al., 2012). A recent human ECoG study reported the propagation velocity of theta waves to be $\sim 2 \mathrm{~m} / \mathrm{s}$ (J. Jacobs, personal communication). This velocity is consistent with our findings and those of the reports of traveling alpha waves observed in the human scalp EEG (Hughes, 1995). The estimated velocity should however be interpreted with caution due to the relatively large distance between the electrodes $(1 \mathrm{~cm})$. The undersampling might result in aliasing artifacts in the spatial domain (Shaw and Roth, 1955; Pocock, 1980). As for the wagon wheel illusion in the temporal domain, both velocity and direction could be misestimated. Indeed, the direction of travel was not consistent over subjects. It is at this stage not clear whether the different travel directions can be explained by spatial undersampling or if it indeed differed over subjects. Furthermore, the placement of the electrode grids was different over subjects, com- plicating the interpretation. Different travel directions have also been observed in other studies (Rémond et al., 1969; Nunez et al., 2001). Future investigations in relation to task parameters are required to uncover the functional role of travel speed or direction. Importantly, it has been demonstrated in EEG recordings that the direction of traveling alpha waves is informative about cognitive performance (Klimesch et al., 2007a; Fellinger et al., 2012; Patten et al., 2012).

In short, we have demonstrated here that bursts of gamma activity propagate over neocortex and the propagating gamma bursts are locked to the phase of traveling alpha waves. Our findings suggest that not only do alpha oscillations serve to coordinate the gamma activity in time, but also in space. In future work to uncover the functional role of this phenomenon would be of great interest.

\section{References}

Alexander DM, Jurica P, Trengove C, Nikolaev AR, Gepshtein S, Zvyagintsev M, Mathiak K, Schulze-Bonhage A, Ruescher J, Ball T, van Leeuwen C (2013) Traveling waves and trial averaging: the nature of single-trial and averaged brain responses in large-scale cortical signals. Neuroimage 73 : 95-112. CrossRef Medline

Bartlett MS (1948) Smoothing periodograms from time-series with continuous spectra. Nature 161:686-687. CrossRef

Bragin A, Jandó G, Nádasdy Z, Hetke J, Wise K, Buzsáki G (1995) Gamma $(40-100 \mathrm{~Hz})$ oscillation in the hippocampus of the behaving rat. J Neurosci 15:47-60. Medline

Canolty RT, Knight RT (2010) The functional role of cross-frequency coupling. Trends Cogn Sci 14:506-515. CrossRef Medline

Canolty RT, Edwards E, Dalal SS, Soltani M, Nagarajan SS, Kirsch HE, Berger MS, Barbaro NM, Knight RT (2006) High gamma power is phaselocked to theta oscillations in human neocortex. Science 313:1626-1628. CrossRef Medline

Cooper R, Winter AL, Crow HJ, Walter WG (1965) Comparison of subcortical, cortical and scal activity using chronically indwelling electrodes in man. Electroencephalogr Clin Neurophysiol 18:217-228. CrossRef Medline

Crick F (1984) Function of the thalamic reticular complex: the searchlight hypothesis. Proc Natl Acad Sci U S A 81:4586-4590. CrossRef Medline

Ermentrout GB, Kleinfeld D (2001) Traveling electrical waves in cortex: insights from phase dynamics and speculation on a computational role. Neuron 29:33-44. CrossRef Medline

Fellinger R, Gruber W, Zauner A, Freunberger R, Klimesch W (2012) Evoked traveling alpha waves predict visual-semantic categorizationspeed. Neuroimage 59:3379-3388. CrossRef Medline

Foxe JJ, Snyder AC (2011) The role of alpha-band brain oscillations as a sensory suppression mechanism during selective attention. Front Psychol 2:154. CrossRef Medline

Freeman WJ, Rogers LJ, Holmes MD, Silbergeld DL (2000) Spatial spectral analysis of human electrocorticograms including the alpha and gamma bands. J Neurosci Methods 95:111-121. CrossRef Medline

Fries P, Nikolić D, Singer W (2007) The gamma cycle. Trends Neurosci 30:309-316. CrossRef Medline

Gunduz A, Brunner P, Daitch A, Leuthardt EC, Ritaccio AL, Pesaran B, Schalk G (2011) Neural correlates of visual-spatial attention in electrocorticographic signals in humans. Front Hum Neurosci 5:89. CrossRef Medline

Hughes JR (1995) The phenomenon of travelling waves: a review. Clin Electroencephalogr 26:1-6. Medline

Jensen O, Kaiser J, Lachaux JP (2007) Human gamma-frequency oscillations associated with attention and memory. Trends Neurosci 30:317324. CrossRef Medline

Jensen O, Bonnefond M, VanRullen R (2012) An oscillatory mechanism for prioritizing salient unattended stimuli. Trends Cogn Sci 16:200-206. CrossRef Medline

Klimesch W, Hanslmayr S, Sauseng P, Gruber WR, Doppelmayr M (2007a) P1 and traveling alpha waves: evidence for evoked oscillations. J Neurophysiol 97:1311-1318. Medline

Klimesch W, Sauseng P, Hanslmayr S (2007b) EEG alpha oscillations: the inhibition-timing hypothesis. Brain Res Rev 53:63-88. CrossRef Medline Lachaux JP, Rodriguez E, Martinerie J, Varela FJ (1999) Measuring phase 
synchrony in brain signals. Hum Brain Mapp 8:194-208. CrossRef Medline

Lakatos P, Shah AS, Knuth KH, Ulbert I, Karmos G, Schroeder CE (2005) An oscillatory hierarchy controlling neuronal excitability and stimulus processing in the auditory cortex. J Neurophysiol 94:1904-1911. CrossRef Medline

Lisman JE, Jensen O (2013) The theta-gamma neural code. Neuron 77: 1002-1016. CrossRef Medline

Llinás R, Ribary U, Contreras D, Pedroarena C (1998) The neuronal basis for consciousness. Philos Trans R Soc Lond Biol 353:1841-1849. CrossRef Medline

Lopes da Silva F (1991) Neural mechanisms underlying brain waves: from neural membranes to networks. Electroencephalogr Clin Neurophysiol 79:81-93. CrossRef Medline

Nunez PL, Srinivasan R (2006) A theoretical basis for standing and traveling brain waves measured with human EEG with implications for an integrated consciousness. Clin Neurophysiol 117:2424-2435. CrossRef Medline

Nunez PL, Wingeier BM, Silberstein RB (2001) Spatial-temporal structures of human alpha rhythms: theory, microcurrent sources, multiscale measurements, and global binding of local networks. Hum Brain Mapp 13: 125-164. CrossRef Medline

O’Keefe J, Recce ML (1993) Phase relationship between hippocampal place units and the EEG theta rhythm. Hippocampus 3:317-330. CrossRef Medline

Oostenveld R, Fries P, Maris E, Schoffelen JM (2011) FieldTrip: open source software for advanced analysis of MEG, EEG, and invasive electrophysiological data. Comput Intell Neurosci 2011:156869. Medline

Osipova D, Hermes D, Jensen O (2008) Gamma power is phase-locked to posterior alpha activity. PLoS One 3:e3990. CrossRef Medline

Patten TM, Rennie CJ, Robinson PA, Gong P (2012) Human cortical traveling waves: dynamical properties and correlations with responses. PLoS One 7:e38392. CrossRef Medline

Petsche H, Rappelsberger P (1970) Influence of cortical incisions on synchronization pattern and travelling waves. Electroencephalogr Clin Neurophysiol 28:592-600. CrossRef Medline
Pocock P V (1980) The spatial and temporal distribution of alpha activity and their modification during motor preparation. In: Rhythmic EEG activities and cortical functioning (Pfurtscheller G, Buser P, Lopes da Silva FH, Petsche H, eds), pp 135-149. Amsterdam: Elsevier.

Rémond A, Lesèvre N, Joseph JP, Rieger H, Lairy GC (1969) The alpha average. I Methodology and description. Electroencephalogr Clin Neurophysiol 26:245-265. CrossRef Medline

Saalmann YB, Kastner S (2011) Cognitive and perceptual functions of the visual thalamus. Neuron 71:209-223. CrossRef Medline

Saalmann YB, Pinsk MA, Wang L, Li X, Kastner S (2012) The pulvinar regulates information transmission between cortical areas based on attention demands. Science 337:753-756. CrossRef Medline

Sato TK, Nauhaus I, Carandini M (2012) Traveling waves in visual cortex. Neuron 75:218-229. CrossRef Medline

Sauseng P, Klimesch W, Heise KF, Gruber WR, Holz E, Karim AA, Glennon M, Gerloff C, Birbaumer N, Hummel FC (2009) Brain oscillatory substrates of visual short-term memory capacity. Curr Biol 19:1846-1852. CrossRef Medline

Shaw JC, Roth M (1955) Potential distribution analysis II. A theoretical consideration of its significance in terms of electrical field theory. Electroencephalogr Clin Neurophysiol 7:285-292. CrossRef Medline

Siegel M, Donner TH, Engel AK (2012) Spectral fingerprints of largescale neuronal interactions. Nat Rev Neurosci 13:121-134. CrossRef Medline

Spaak E, Bonnefond M, Maier A, Leopold DA, Jensen O (2012) Report layer-specific entrainment of gamma-band neural activity by the alpha rhythm in monkey visual cortex. Curr Biol 22:2313-2318. CrossRef Medline

Tort AB, Komorowski R, Eichenbaum H, Kopell N (2010) Measuring phase-amplitude coupling between neuronal oscillations of different frequencies. J Neurophysiol 104:1195-1210. CrossRef Medline

Voytek B, Canolty RT, Shestyuk A, Crone NE, Parvizi J, Knight RT (2010) Shifts in gamma phase-amplitude coupling frequency from theta to alpha over posterior cortex during visual tasks. Front Hum Neurosci 4:9. CrossRef Medline 